\title{
Modélisation et simulation d'un système de suivi d'un mini heliostat
}

\section{Marie Pascaline SARR ${ }^{1, *}$, Ababacar THIAM ${ }^{1,3}$, Biram DIENG $^{2}$, Fallou NDIAYE $^{3}$}

${ }^{1}$ Groupe de recherche Efficacité et Systèmes Energétiques, Université Alioune Diop de Bambey, Sénégal

${ }^{2}$ Groupe de Recherche Energies Renouvelables, Matériaux et Laser, Université Alioune Diop de Bambey, Sénégal

${ }^{3}$ Laboratoire d'Energétique Appliquée, Ecole Supérieure Polytechnique Dakar, Sénégal

․mariepascaline.sarr@uadb.edu.sn

\section{INFOS SUR L'A R T I C L E}

Historique de l'article:

Reçu le : 31 juillet 2019

Reçu en format revisé le : 26 novembre 2019

Accepté le : 29 novembre 2019

Mots-Clés:suivi solaire, mini héliostat,

monture altazimutale, moteurs pas à pas

\section{RE S U M E}

Dans ce présent article il s'agit de la mise en place d'un système de suivi d'un mini héliostat basé sur des équations astronomiques. Deux moteurs pas à pas bipolaires à aimant permanent assurent le suivi solaire du mini héliostat suivant la hauteur et l'azimut. Des simulations du système de suivi et des moteurs sont réalisées dans Matlab/Simulink pour les jours d'équinoxe et de solstice. Les résultats obtenus ont permis d'analyser la plage de mouvement du mini héliostat en fonction de ces différents jours.

\section{INTRODUCTION}

Aujourd'hui, l'un des principaux défis pour l'Afrique est de satisfaire les besoins énergétiques de sa population qui ne cesse de croitre. Parmi les $14 \%$ de la population mondiale n'ayant pas accès à l'électricité, $56 \%$ proviennent de l'Afrique Subsaharienne et pour la plupart il s'agit des populations vivant en milieu rural (Montaigne, 2019). L'exploitation du potentiel solaire dont dispose cette région de l'Afrique (environ 5.5 $\mathrm{kWh} / \mathrm{m} /$ jour) permettrait de répondre aux besoins énergétiques de la population (Azoumah et al., 2010). De toutes les technologies solaires thermodynamiques, les centrales à tours sont identifiées comme étant la technologie la plus efficace pour capter et convertir l'énergie solaire en chaleur puis en électricité (Bhargava et al., 2014). Toutefois, le coût élevé des centrales à tours dont $50 \%$ consacré aux héliostats (Kolb et al., 2007), constitue un obstacle à l'essor de cette technologie surtout dans les pays en voie de développement. De plus les compétences requises pour l'installation de telles centrales ne sont pas bien réparties dans toute l'Afrique (N'Tsoukpoe, et al., 2016). La mise en place de mini centrales à tours avec de mini héliostats permet de réduire le coût de ces centrales et de faciliter leur conception dans la sousrégion. Les performances des centrales à tours dépendent fortement de l'efficacité du champ solaire qui, à son tour, est liée aux systèmes de suivi et de contrôle. Le suivi de l'héliostat est identifié comme étant l'un des domaines de recherche à poursuivre pour rendre les centrales à tours beaucoup plus compétitives (Hafeza et al., 2018). Ainsi la maitrise du suivi des héliostats est essentielle pour la mise en place de minicentrales à tours en Afrique Subsaharienne. L'objectif de ce travail est de mettre en place un système de suivi solaire automatique basé sur des équations astronomiques pour un mini héliostat de monture Alt azimutale.

\section{MÉTHODOLOGIE}

Les héliostats sont des miroirs mobiles motorisés qui suivent le soleil suivant deux axes afin de réfléchir les rayons solaires au sommet d'une tour où se trouve le récepteur. Le suivi des héliostats se fait généralement en boucle ouverte avec des équations astronomiques qui est beaucoup moins onéreuse que celle en boucle fermée (Pisirira, et al., 2016). Ainsi pour pouvoir capter le maximum d'énergie solaire, il est nécessaire de conceptualiser le mouvement du soleil tout au long de la journée. A la suite de la modélisation de la trajectoire du soleil, des modèles mathématiques régissant les angles de suivi du mini héliostat sont établis. Les moteurs pas à pas utilisés sont ensuite modélisés.

\subsection{Modélisation de la position du soleil et du mini héliostat}

La position du soleil varie suivant la latitude du lieu $\varphi$, l'angle horaire $\omega$ et la déclinaison $\delta$. Elle est modélisée par deux angles à savoir la hauteur $\alpha$ et l'azimut A représentés au niveau de la figure 1. Ces deux angles sont obtenus grâce aux équations (1) et (2) (Chong et al., 2012) :

$\alpha=\sin ^{-1}(\sin \delta \sin \phi+\cos \delta \cos \omega \cos \phi)$

(1)

$A=\cos ^{-1}\left(\frac{\sin \delta \cos \phi-\cos \delta \cos \omega \sin \phi}{\cos \alpha}\right)$ 




Fig.1. Repérage du soleil en fonction des coordonnées géographiques

Le mini héliostat suit le soleil suivant la hauteur et l'azimut. Il est représenté au niveau de la figure 2.



Fig.2. Héliostat de monture azimut élévation

La position du mini héliostat par rapport à la tour est défini par deux angles à savoir l'angle focal $\lambda$ et l'angle de face $\varphi$ représentés sur la figure 3 .



Fig3: Position du mini héliostat par rapport à la tour et à la position du soleil (Salgado-Plasencia, et al., 2019)

L'angle d'incidencecaractérise l'angle formé par la normale de l'héliostat et le vecteur soleil ou le récepteur. Il est défini par l'équation suivante :

$\theta=\frac{1}{2} \cos ^{-1}(\sin \alpha \sin \lambda+\cos \alpha \sin A \cos \lambda \sin \phi$
$+\cos \alpha \cos A \cos \lambda \cos \phi)$

La variation de l'angle d'incidence suivant la position de l'héliostat au niveau du champ héliostatique est traduite par les angles de la hauteur $\alpha_{\mathrm{AE}}$ et de l'azimut $\rho_{\mathrm{AE}}$ du mini héliostats obtenus par les équations suivantes :

$$
\begin{aligned}
& \alpha_{A E}=\sin ^{-1}\left(\frac{\sin \lambda+\sin \alpha}{2 \cos \theta}\right) \\
& \cos \rho_{A E}=\frac{\cos \alpha \cos A+\cos \lambda \cos \varphi}{2 \cos \theta \cos \alpha_{A E}}
\end{aligned}
$$

Si $\cos \rho_{A E}>0$

$\rho_{A E}^{+}=\sin ^{-1}\left(\frac{\cos \alpha \sin A+\cos \lambda \sin \varphi}{2 \cos \theta \cos \alpha_{A E}}\right)$

Si $\cos \rho_{A E}<0, \rho_{A E}^{-}=\pi-\rho_{A E}^{+}$

L'angle d'azimut $\rho_{\mathrm{AE}}$ est négatif en début de journée lorsque le soleil est à l'est, nul à midi solaire et positif le soir quand le soleil est à l'Ouest. La position repos de l'héliostat représente la position $\left(\alpha_{\mathrm{AE}}=90, \rho_{\mathrm{AE}}\right.$ $=0$ ). Dans ce cas l'héliostat est orienté plein Sud avec le miroir parallèle à l'axe

Pour assurer le suivi du mini héliostat suivant la hauteur et l'azimut, deux moteurs pas à pas bipolaires à aimant permanent sont mis en place. Les moteurs pas à pas en raison de leur précision sont très utilisés pour le suivi en boucle ouverte (Malan, 2014). Leur modélisation est effectuée dans le paragraphe suivant.

\subsection{Modélisation du moteur pas à pas}

Le moteur pas à pas bipolaire est modélisé par les quatre équations suivantes :

$$
\begin{aligned}
& \frac{d i_{a}}{d t}=u_{a}+K_{m} \omega \sin N \theta_{p}-R i_{a} L \\
& \frac{d i_{b}}{d t}=u_{b}+K_{m} \omega \sin N \theta_{p}-R i_{b} L \\
& \frac{d \omega}{d t}=K_{m} i_{b} \cos N \theta_{p}-T_{L}-K_{m} i_{a} \sin N \theta-K_{v} \omega J \\
& \frac{d \theta_{p}}{d t}=\omega
\end{aligned}
$$

Avec $\mathrm{K}_{\mathrm{m}}, \mathrm{N}$ et $\mathrm{R}$ représentant respectivement la constante de couple, le nombre de conducteurs de l'enroulement d'induit et la résistance interne.

Ces quatre équations tiennent compte des deux bobines du stator $\left(i_{\mathrm{a}}\right.$ et $\left.\mathrm{i}_{\mathrm{b}}\right)$ pour le déplacement du rotor, de la vitesse de rotation $(\omega)$ et de la position $\left(\theta_{\mathrm{p}}\right)$.

Avec $\theta_{\mathrm{p}}=\rho_{\mathrm{AE}}$ suivant l'azimut

et $\theta_{\mathrm{p}}=\alpha_{\mathrm{AE}}$ suivant la hauteur

Ceci va permettre de réaliser le schéma bloc du moteur pas à pas sous Simulink. Les caractéristiques des moteurs sont présentées au niveau du tableau suivant.

Table 4.Caractéristiques du moteur

\begin{tabular}{|l|c|}
\hline \multicolumn{1}{|c|}{ Paramètres } & Spécifications \\
\hline Courant nominal & $1,68 \mathrm{~A}$ \\
\hline Résistance par phase & $1.65 \Omega$ \\
\hline Inductance par phase & $2,8 \mathrm{mH}$ \\
\hline Inertie du moteur & $7.510^{-4} \mathrm{~kg} . \mathrm{m}$ \\
\hline Coefficient de viscosité & $0.001 \mathrm{~N} . \mathrm{m} . \mathrm{s} / \mathrm{rad}$ \\
\hline
\end{tabular}




\section{RESULTATS ET DISCUSSIONS}

Les variations horaires des angles caractéristiques d'un héliostat de monture Alt azimutale ont été étudiées numériquement pour les jours du 21 Décembre (solstice d'hiver), 21 Mars (équinoxe de printemps) et 21 Juin (solstice d'été). De telles variations peuvent être obtenues pour n'importe quel jour de l'année en utilisant la procédure numérique développée dans cette recherche. L'héliostat est simulé avec un angle focal $\lambda=45^{\circ}$, un angle de face $\varphi=0^{\circ}$ (l'héliostat est au Nord de la tour) une hauteur de $1,5 \mathrm{~m}$. La figure 4 représente les courbes de variation de l'angle d'incidence pour un héliostat se trouvant au nord de la tour.



Fig.4: Variation de l'angle d'incidence en fonction $d u$ jour

La variation de l'angle d'incidence est beaucoup plus élevée pour le jour du 21 Décembre quand le soleil est au plus bas. Cette variation est faible pour le jour du 21 Juin jour où la hauteur du soleil est plus élevée. Toutefois la courbe de variation de l'angle d'incidence est différente suivant la position de l'héliostat $(\lambda$ et $\varphi)$ au niveau du champ.

Les figures 5 et 6 présentent les variations de la hauteur et de l'azimut du mini héliostat pour les mêmes jours.



Fig5: Variation de la hauteur du mini héliostat suivant le jour

La hauteur du mini héliostat est plus importante pour le jour du 21 Juin et est moindre pour le 21 Décembre. Cette variation de la hauteur du mini héliostat est donc proportionnelle à celle de la hauteur $\mathrm{du}$ soleil qui est maximum le jour du 21 Juin (solstice d'été).



Fig.6: Variation de l'azimut du mini héliostat en fonction du jour

L'azimut du mini héliostat est plus important le jour du 21 Décembre et est faible pour le jour du 21 Juin. Cette courbe de variation de l'azimut du mini héliostat dépend fortement de la position du mini héliostat par rapport à la tour et des coordonnées géographiques.

Cependant le temps de suivi au niveau de l'azimut et de la hauteur est plus important le jour du 21 Juin (jour le plus long) que les autres jours de l'année peu importe la position du mini héliostat. Ainsi ce jour est pris comme référence pour le choix des paramètres de simulations des moteurs.

Les figures 7 et 8 traduisent les courbes de variation de la tension au niveau des deux bobines du stator. Un décalage de $90^{\circ}$ est observé entre les deux sources. Ceci traduit l'alternance des deux sources forçant ainsi le rotor à se déplacer.



Fig.7: Courbe de la tension d'alimentation $U_{a} d u$

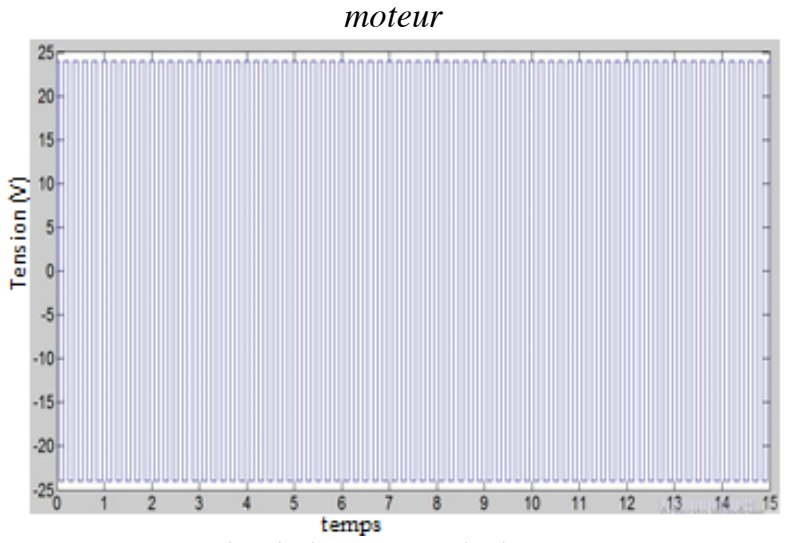

Fig.8: Courbe de la tension d'alimentation $U_{b} d u$ moteur

Les figures 9 et 10 présentent respectivement les variations de la vitesse et de la position du moteur pas 
à pas bipolaire à aimant permanent obtenues avec la modélisation du moteur pas à pas.

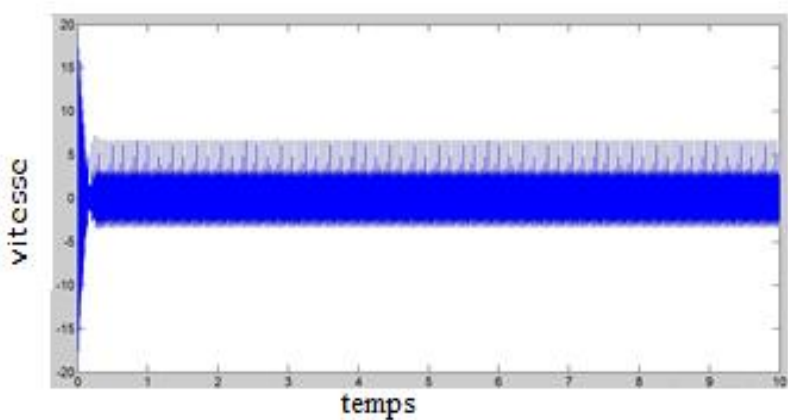

Fig.9:Vitesse de rotation du moteur pas à pas

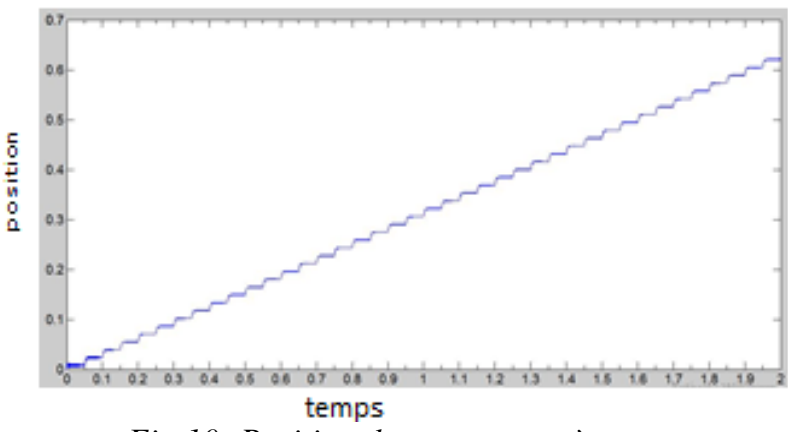

Fig.10: Position du moteur pas à pas

Au niveau de la courbe de variation de la vitesse de rotation une petite oscillation est observée tout au début avant de se stabiliser. La faible fréquence de commande influe sur la vitesse de rotation du moteur permettant au moteur de tourner autour de la même valeur. La position du moteur pas à pas varie à partir de 0 pour croître jusqu'à une certaine valeur traduisant ainsi le déplacement à sens unique du moteur (Est vers Ouest) au niveau de l'azimut.

\section{CONCLUSION}

Le système de suivi du mini héliostat de monture alt azimutale est effectué en se basant sur l'heure et les coordonnées géographiques. La variation des jours influe sur la plage de fonctionnement des héliostats. Ainsi pour n'importe quel jour de l'année, il est possible d'obtenir la plage de mouvement du mini héliostat pour un site donné. En perspectives, il est envisagé de mettre en place un système de suivi en boucle fermée pour un suivi plus précis.

\section{REMERCIEMENTS}

Tous nos remerciements au Centre d'Excellence Africain de Mathématiques et Technologie de l'Information et de la Communication de Saint Louis, Sénégal et à World Federation of Scientist,

\section{REFERENCES}

Azoumah Y., et al., 2010. Siting guidelines for concentrating solar power plants in the Sahel: Case study of Burkina Faso. Solar Energy, 84, 15451553.

Bhargava K. R. , et al.,2014. Life Cycle cost optimized heliostat size for power towers. Energy Procedia 49, $40-49$.
Chong K. K. and Tan M. H., 2012. Comparison Study of Two Different Sun-Tracking Methods in Optical Efficiency of Heliostat Field. International Journal of Photenergy, 1908364, p. 10.

Hafeza A., Yousefa A. et Haraga N., 2018. Solar tracking systems: technologies and trackers drive types- A review. Renewable and Sustainable Energy Reviews, 91, 754-782.

Kolb G. [et al.] Heliostat cost reduction study, [Revue] // Sandia National Laboratories, USA. 2007.

Malan K., 2014. A Heliostat Field Control System Rapport de l'Université de Stellenbosch, South Africa, p. 129.

Montaigne Institut, 2019. Énergie solaire en Afrique : un avenir rayonnant? [Rapport].

N'Tsoukpoe Kokouvi Edem et al., 2016. Integrated design and construction of a micro-central tower power plant. Energy for Sustainable Development, . $31,1-13$.

Pisirira O.M. et Bingölb O., 2015. Industrial PC Based Heliostat Control for Solar Power Towers. ACTA PHYSICA POLONICA A Special issue of the 2nd International Conference on Computational and Experimental Science and Engineering (ICCESEN 2015), Vol.130, 36-40.

Salgado-Plasencia Eugenio et al., 2019. SCADABased Heliostat Control System with a Fuzzy Logic Controller for the Heliostat Orientation. Applied Sciences, 9, 29-66 